\title{
Estructura de tallas, condición reproductiva, relación longitud-peso y biomasa de Cryphiops caementarius (Decapoda: Palaemonidae) en los ríos de la costa del Perú
}

\author{
Size structure, reproductive condition, length-weight relationship and biomass of \\ Cryphiops caementarius (Decapoda: Palaemonidae) in Peruvian coastal rivers
}

\author{
Stive Flores-Gómez ${ }^{1,2}$
}

\section{Resumen}

\begin{abstract}
El objetivo de este estudio fue determinar la estructura poblacional, proporción de sexos, condición reproductiva, índice poblacional y biomasa de Cryphiops caementarius en los ríos Cañete (Lima), Tambo, Ocoña y Majes (Arequipa), Perú. La investigación se efectuó en base a información obtenida por el Instituto del Mar del Perú. El análisis biológico-poblacional se efectuó a partir de muestras de camarón obtenidas por capturas sistemáticas en diferentes estratos altitudinales. Los parámetros de calidad del agua presentaron valores que estuvieron dentro de los rangos aceptables para el normal desarrollo del recurso. Las tallas modales del recurso fueron inferiores a $70 \mathrm{~mm}$ (talla mínima de captura) en los ríos Cañete, Tambo y Majes. Se determinó mayor proporción de machos en los ríos Cañete, Tambo y Ocoña, y menor en el río Majes. La proporción de hembras en desove fue inferior al 10\% en los meses evaluados (julio-diciembre de 2019). El coeficiente de crecimiento relativo del camarón fue superior en los machos respecto a las hembras en los ríos Tambo, Ocoña y Majes, y similar en machos y hembras en el río Cañete. Majes fue el río con mayor biomasa con relación a los otros ríos y, por lo tanto, el más productivo. La alta biomasa en los diferentes estratos altitudinales de los ríos estuvo asociada
\end{abstract}

\footnotetext{
${ }_{1}^{1}$ Área de Investigaciones en Recursos de Aguas Continentales, Instituto del Mar del Perú, Callao, Perú

${ }^{2}$ E-mail:danstive@gmail.com
}

Recibido: 21 de octubre de 2020

Aceptado para publicación: 30 de abril de 2021

Publicado: 24 de agosto de 2021

CLos autores. Este artículo es publicado por la Rev Inv Vet Perú de la Facultad de Medicina Veterinaria, Universidad Nacional Mayor de San Marcos. Este es un artículo de acceso abierto, distribuido bajo los términos de la licencia Creative Commons Atribución 4.0 Internacional (CC BY 4.0) [https:// creativecommons.org/licenses/by/4.0/deed.es] que permite el uso, distribución y reproducción en cualquier medio, siempre que la obra original sea debidamente citada de su fuente original 
a las altas concentraciones de oxígeno y dureza total del agua. La información de esta investigación es de valor para direccionar las estrategias de ordenación pesquera sostenible.

Palabras clave: calidad del agua, pesquería, biomasa, reproducción, población

\section{Abstract}

The aim of this study was to determine the population structure, sex ratio, reproductive condition, population index and biomass of Cryphiops caementarius in the Cañete (Lima), Tambo, Ocoña and Majes (Arequipa) rivers, Peru. The research was carried out based on information obtained by the Instituto del Mar del Perú. The biologicalpopulation analysis was carried out from prawn samples obtained by systematic captures in different altitude strata. The water quality parameters presented values that were within the acceptable ranges for the normal development of the resource. The modal sizes of the resource were less than $70 \mathrm{~mm}$ (minimum catch size) in the Cañete, Tambo and Majes rivers. A higher proportion of males was determined in the Cañete, Tambo and Ocoña rivers, and a lower proportion in the Majes river. The proportion of spawning females was less than 10\% in the evaluated months (July-December 2019). The relative growth coefficient of prawns was higher in males than in females in the Tambo, Ocoña and Majes rivers, and similar in males and females in the Cañete river. The Majes was the river with the highest biomass in relation to the other rivers and, therefore, the most productive. The high biomass in the different altitudinal strata of the rivers was associated with high concentrations of oxygen and total hardness of the water. The information from this research is of value in guiding sustainable fisheries management strategies.

Key words: water quality, fishery, biomass, reproduction, population

\section{INTRODUCCIÓN}

Camarón es el nombre común de varias especies que habitan los ríos de la vertiente occidental de los Andes del Perú, e involucra a los géneros Cryphiops, Macrobrachium, Palaemon y Atya. Dada su abundancia, Cryphiops caementarius, que es migratoria (Valle y Kuroiwa, 2010), es la más representativa y permite el sostenimiento de la pesquería artesanal en los ríos de la costa centro-sur del Perú (Meruane et al., 2006) y de la costa norte de Chile (Morales y Meruane, 2013).
Aproximadamente el $80 \%$ de la biomasa del camarón existente en la costa peruana se encuentra en los ríos Ocoña, Majes-Camaná y Tambo (Arequipa), donde la actividad extractiva es intensa (Yépez, 2009). Sin embargo, también se extrae en el río Cañete (Baltazar y Colan, 2014), que se ubica en la Región Lima, pero en menores volúmenes (Zacarías y Yépez, 2015).

El objetivo de este estudio fue evaluar la población $C$. caementarius en los ríos Cañete, Tambo, Ocoña y Majes-Camaná, a fin de contribuir con información científica actualizada sobre el estado del recurso para fines de conservación y manejo sostenible. 


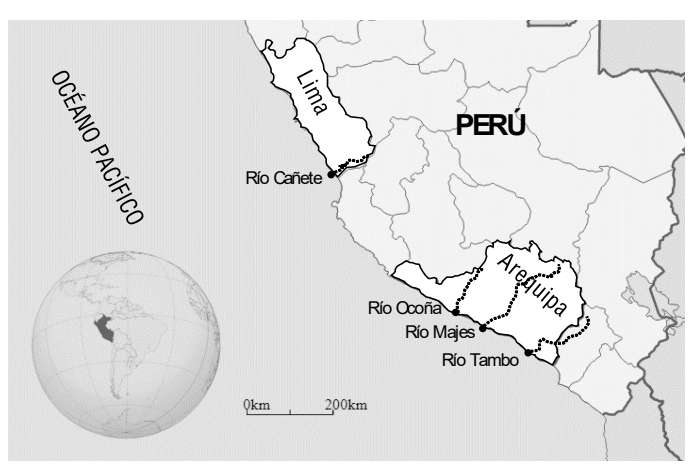

Figura 1. Ámbito de estudio de $C$. caementarius (adaptado de wikipedia.org)

\section{Materiales y Métodos}

\section{Ámbito de Estudio}

Los datos del estudio fueron obtenidos por el Instituto del Mar del Perú entre julio y diciembre de 2019, y corresponden a los ríos Cañete, Majes-Camaná, Ocoña y Tambo, el primero ubicado en la región Lima y los siguientes en la región Arequipa, Perú. En el trabajo de campo se registraron parámetros fisicoquímicos del agua y de faenas de pesca, los cuales se ejecutaron en áreas de los siguientes rangos altitudinales: río Cañete: 01 $800 \mathrm{msnm}$; río Tambo: 01-400 msnm; río Ocoña: 01-700 msnm y río Majes-Camaná (en adelante considerando como Majes): 01 1000 msnm (Figura 1).

\section{Información Limnológica}

Se determinaron los parámetros de temperatura del agua (medición con termómetro protegido, precisión al $0.1^{\circ} \mathrm{C}$ ), temperatura ambiental (termómetro ambiental), oxígeno disuelto (oxímetro digital), cloruro (kit Hach) y dureza total (kit Hach), para analizar la relación recurso-ambiente, considerando las diferencias que cada especie tiene con su entorno (Parada et al., 2013).
Las muestras de agua fueron colectadas del nivel superficial en orilla utilizando un frasco muestreador y el análisis se efectuó in situ (Autoridad Nacional del Agua [ANA], 2016). Asimismo, se registró la presencia de brazos y meandros en las zonas de muestreo. Se efectuaron análisis de correlación de Pearson entre los parámetros fisicoquímicos del agua y las biomasas del camarón para cada río.

\section{Pesca Experimental}

La colecta de camarón se efectuó mediante faenas de pesca utilizando el método de «buceo a pulmón» en estaciones determinadas para cada uno de los ríos. La operación de pesca consideró «barridos» en contra corriente efectuado por ocho extractores experimentados que efectuaron dos recorridos en transectos de $40 \mathrm{~m}$. Cada barrido demandó cerca de 20 minutos, dependiendo del tipo de fondo y del caudal. Las capturas de cada pescador fueron pesadas al gramo utilizando una balanza portátil (precisión $0.01 \mathrm{~g}$ ) y se hizo el conteo de los individuos obtenidos por transecto; asimismo, se anotó la cantidad de ejemplares que no pudieron ser atrapados («escapes»), información dada por las personas que recolectaron los especímenes, para estimar la eficiencia de la pesca y los índices de abundancia (Yépez y Bandín, 1996).

\section{Muestreo Biométrico y Biológico}

Se efectuó el muestreo de 60 ejemplares del total de los capturados en cada estrato altitudinal. Los muestreos se efectuaron siguiendo la metodología de Yépez y Bandín (1996). Se consideró el registro de la longitud total $(\mathrm{mm})$, longitud del cefalotórax $(\mathrm{mm})$, peso total $(\mathrm{g})$, peso abdominal $(\mathrm{g})$, sexo y madurez gonadal de cada ejemplar de la muestra. La medición de ejemplares se realizó con un vernier mecánico y los pesos con 
una balanza electrónica portátil de $0.01 \mathrm{~g}$ de precisión. Los individuos fueron medidos al milímetro y separados en clases o intervalos de $5 \mathrm{~mm}$ a la unidad inmediata inferior.

La información obtenida se utilizó para determinar las principales características biométricas y biológicas del recurso, tales como la estructura de tallas (Csircke, 1980), proporción sexual, condición reproductiva (Yépez y Bandín, 1996) y relación longitud peso (García et al., 2009). La determinación de la condición reproductiva se efectuó mediante el análisis macroscópico de las gónadas según la escala de madurez gonadal utilizada por (Yépez y Bandín, 1996).

En el análisis de la proporción sexual teórica 1 macho: 1 hembra se utilizó la prueba estadística Chi cuadrado $(\alpha=0.05)$, considerándose como significativo a $\mathrm{X}^{2}>3.84$ (valor de tabla: 1 grado de libertad).

\section{Aspectos Poblacionales}

La biomasa se estimó por el método modificado de «área barrida», que considera la densidad del recurso determinada a partir de la captura por área (o sector) barrida efectiva y de la extrapolación a la totalidad del área de cada estrato (Espino y WosnitzaMendo, 1984). El método implicó la determinación del número y peso de ejemplares capturados por extractor (incluyendo los escapes) en cada sección, para estimar las densidades, concentración y población en número y biomasa por estratos del río. Se determinaron los índices de concentración (densidad y biomasa media), la abundancia en número y biomasa por estratos (Wasiw y Yépez, 2017).

Para determinar el área del espejo de agua de cada estrato, clave para las estimaciones poblacionales, se multiplicó la longitud del curso (según cálculo cartográfico) por el ancho medio del lecho; adicionando factores de sinuosidad (meandro) y morfometría (ramal o brazo) para ajustar la estimación del área.
Resultados

\section{Parámetros Fisicoquímicos de Calidad del Agua}

Según los resultados (Cuadro 1), la temperatura del agua incrementó de julio (19.1 ${ }^{\circ} \mathrm{C}$ media en el río Cañete) a diciembre (25.1 ${ }^{\circ} \mathrm{C}$ media en el río Majes). La concentración media de oxígeno disuelto fluctuó sobre 10 $\mathrm{mg} / \mathrm{l}$ en los cuatro ríos. Respecto a la dureza total media, el río Tambo presentó $453.7 \mathrm{mg} / \mathrm{l}$, valor superior al de los otros ríos, evidencia que es un río cuya agua es rica en carbonatos. La concentración media de cloro fue mayor en el río Majes $(41.0 \mathrm{mg} / \mathrm{l})$ respecto a los otros ríos, siendo la concentración más baja en el río Tambo $(35.2 \mathrm{mg} / \mathrm{l})$.

\section{Estructura Poblacional por Talla}

C. caementarius (Figura 2) tuvo características particulares en cada río. Las tallas en el río Cañete variaron entre 30 a $115 \mathrm{~mm}$ con moda $60 \mathrm{~mm}$, en Ocoña de 35 a $130 \mathrm{~mm}$ con moda $70 \mathrm{~mm}$, en Majes de 35 a $145 \mathrm{~mm}$ con moda $60 \mathrm{~mm}$ y en Tambo de 35 a 130 mm con moda $65 \mathrm{~mm}$. Los resultados muestran que la moda en los ríos Cañete, Tambo y Majes fue inferior a la talla mínima de captura (TMC) de $70 \mathrm{~mm}$, establecida en la Resolución Ministerial N. ${ }^{\circ}$ 209-2001-PRODUCE.

El análisis de tallas por estratos altitudinales (Figura 3), permitió observar que, en todos los estratos altitudinales evaluados del río Cañete, la moda fue inferior a la TMC. En el río Tambo, en los estratos de 201-300 y 301-400 msnm, la moda superó la TMC y se mantuvo por debajo en los dos primeros estratos (01-100 y 101-200 msnm). En el río Ocoña, la moda fue inferior a la TMC solo en los dos primeros estratos, mientras que en el río Majes, la moda fue inferior a la TMC en los primeros cinco estratos altitudinales y superior en los siguientes cinco. 
Cuadro 1. Resumen los parámetros fisicoquímicos del agua de cuatro ríos de la costa peruana

\begin{tabular}{lllcccc}
\hline Parámetro & Río & Mes & Mín. & Máx. & Media & D.E. \\
\hline Temperatura & Cañete & jul-19 & 17.0 & 20.4 & 19.1 & 1.0 \\
$\left({ }^{\circ} \mathrm{C}\right)$ & Tambo & sep-19 & 18.0 & 23.1 & 20.3 & 1.7 \\
& Ocoña & nov-19 & 19.8 & 24.1 & 21.9 & 1.2 \\
& Majes & dic-19 & 19.9 & 28.9 & 25.1 & 2.3 \\
Oxígeno & Cañete & jul-19 & 9.1 & 12.4 & 10.2 & 0.7 \\
disuelto, $\mathrm{O}_{2}$ & Tambo & sep-19 & 9.1 & 10.5 & 10.0 & 0.4 \\
$(\mathrm{mg} / \mathrm{l})$ & Ocoña & nov-19 & 8.0 & 10.2 & 9.4 & 0.6 \\
& Majes & dic-19 & 7.7 & 13.1 & 10.3 & 1.6 \\
Dureza total, & Cañete & jul-19 & 165.0 & 234.0 & 203.6 & 13.7 \\
$\mathrm{CaCO}(\mathrm{mg} / \mathrm{l})$ & Tambo & sep-19 & 393.3 & 530.1 & 453.7 & 31.6 \\
& Ocoña & nov-19 & 119.7 & 188.1 & 148.2 & 16.5 \\
& Majes & dic-19 & 171.0 & 393.3 & 268.5 & 77.4 \\
$\mathrm{Cloruro}_{3} \mathrm{Cl}$ & Cañete & jul-19 & 20.0 & 60.0 & 35.2 & 11.2 \\
$(\mathrm{mg} / \mathrm{l})$ & Tambo & sep-19 & 20.0 & 50.0 & 29.3 & 11.0 \\
& Ocoña & nov-19 & 10.0 & 60.0 & 37.5 & 13.6 \\
& Majes & dic-19 & 20.0 & 60.0 & 41.0 & 11.8 \\
\hline
\end{tabular}

Cuadro 2. Proporción sexual de Cryphiops caementarius en los ríos de la costa centro y sur del Perú

\begin{tabular}{|c|c|c|c|c|}
\hline Río & $\widehat{\partial} \sigma^{\lambda}$ & 우 & $\begin{array}{c}\text { Proporción } \\
\delta^{\lambda} \delta^{\lambda}:+q\end{array}$ & $X^{2}$ \\
\hline Cañete & 685 & 193 & $3.5: 1.0$ & $275.7 *$ \\
\hline Tambo & 550 & 312 & $1.8: 1.0$ & $65.7 *$ \\
\hline Ocoña & 788 & 627 & $1.3: 1.0$ & $18.3^{*}$ \\
\hline Majes & 864 & 996 & $0.9: 1.0$ & $9.4^{*}$ \\
\hline
\end{tabular}

\section{Proporción de Sexos}

Los resultados (Cuadro 2) evidencian una mayor proporción de machos respecto a hembras en los ríos Cañete, Tambo y Ocoña; mientras que en el río Majes se observó un predominio de hembras. En todos los casos hubo diferencias significativas en la proporción de machos y hembras $(\mathrm{p}<0.05)$.

\section{Condición Reproductiva}

Se observó predominancia $(>75 \%)$ de hembras en fase gonadal II (maduración incipiente) en ambos sexos para el periodo julio-diciembre 2019. El porcentaje de machos en fase gonadal III (madurante avanzado) fue menor al $2.5 \%$ en los ríos Cañete, Tambo y Ocoña, y menor al 14\% en el río Majes, mientras que, en el caso de hembras en fase III (madurante intermedio) varió entre $7.3 \mathrm{y}$ $11.6 \%$, y en fase IV (desove) varió de 5.1 a $8.7 \%$ (Cuadro 3). 
Cuadro 3. Madurez gonadal (\%) de Cryphiops caementarius en ríos de la costa centro y sur del Perú

\begin{tabular}{|c|c|c|c|c|c|c|c|c|}
\hline \multirow{2}{*}{$\begin{array}{l}\text { Estadio de } \\
\text { madurez }\end{array}$} & \multicolumn{2}{|c|}{ Cañete } & \multicolumn{2}{|c|}{ Tambo } & \multicolumn{2}{|c|}{ Ocoña } & \multicolumn{2}{|c|}{ Majes } \\
\hline & ठิ & $q q$ & $\hat{\sigma}$ & 우 & ठ઼ & 우 & $\hat{\sigma} \tilde{O}$ & 우우 \\
\hline I & 0 & 0 & 0.2 & 0 & 0 & 0 & 0 & 0 \\
\hline II & 99.6 & 87.6 & 99.1 & 84.3 & 97.8 & 83.4 & 86.6 & 79.6 \\
\hline III & 0.4 & 7.3 & 0.7 & 10.6 & 2.2 & 7.8 & 13.4 & 11.6 \\
\hline IV & 0 & 5.2 & 0 & 5.1 & 0 & 8.8 & 0 & 8.7 \\
\hline $\mathrm{V}$ & 0 & 0 & 0 & 0 & 0 & 0 & 0 & 0 \\
\hline
\end{tabular}

I: inmaduro: II: maduración incipiente (machos), intermedia (machos); III: maduración intermedia (hembras), avanzada (machos); IV: maduración avanzada (hembras), post expulsante (machos); V: Posdesove (hembras)

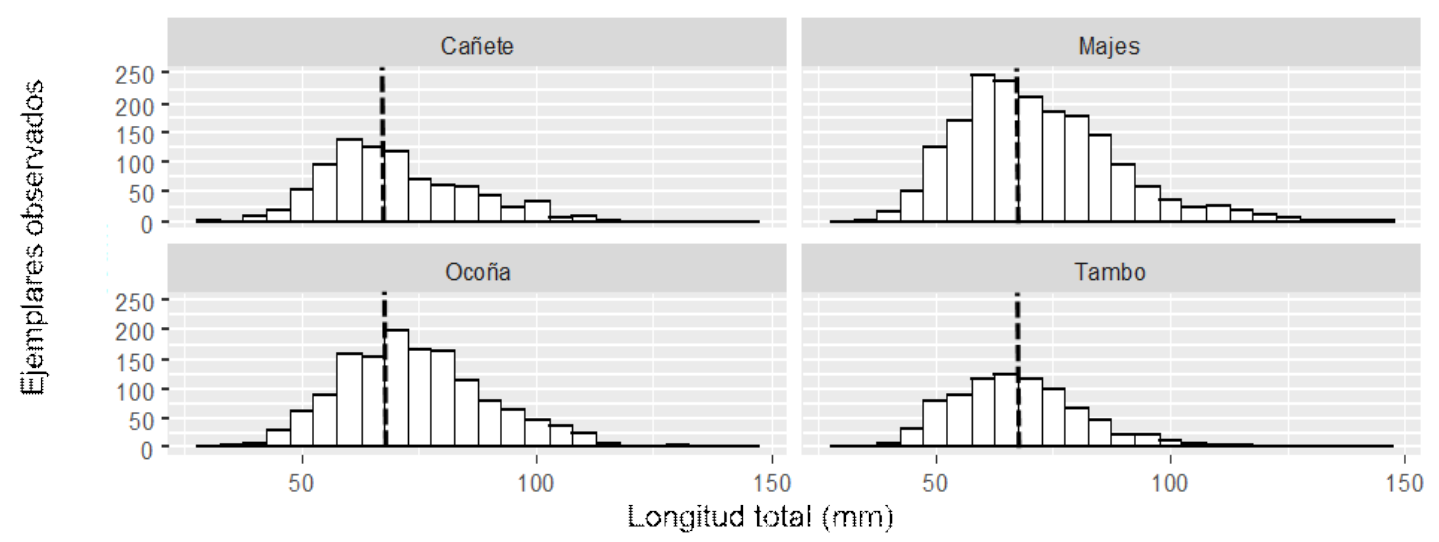

Figura 2. Estructura de tallas de Cryphiops caementarius en cuatro ríos del Perú. Línea vertical segmentada es la talla mínima de captura (TMC)

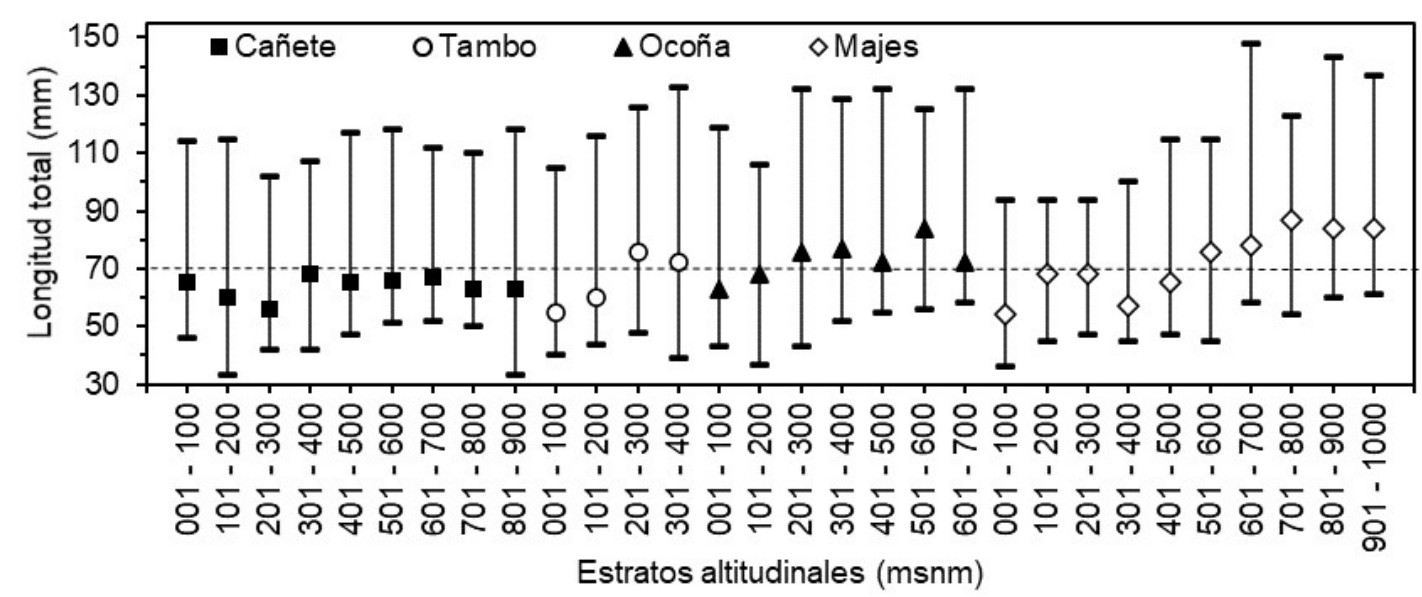

Figura 3. Variación altitudinal de las tallas mínimas, máximas y modales de Cryphiops caementarius en cuatro ríos de la costa peruana. Las formas (cuadrado, círculo, triángulo y rombo) son las modas; línea horizontal segmentada es la talla mínima de captura - TMC (70 mm); bigotes superior e inferior indican las tallas máxima y mínima 
Cuadro 4. Resumen de los parámetros de la regresión lineal entre la longitud y el peso de Cryphiops caementarius en los ríos Cañete, Tambo, Ocoña y Majes, julio-diciembre 2019

\begin{tabular}{|c|c|c|c|c|}
\hline Río & Parámetro & के & 우 & Total \\
\hline \multirow[t]{4}{*}{ Cañete } & $a$ & -11.39 & -11.36 & -11.48 \\
\hline & $b$ & 3.23 & 3.22 & 3.25 \\
\hline & $\mathrm{R}^{2}$ & 0.98 & 0.96 & 0.98 \\
\hline & $\mathrm{N}$ & 685 & 193 & 878 \\
\hline \multirow[t]{4}{*}{ Tambo } & $a$ & -11.35 & -10.91 & -11.42 \\
\hline & $b$ & 3.24 & 3.12 & 3.25 \\
\hline & $\mathrm{R}^{2}$ & 0.97 & 0.98 & 0.96 \\
\hline & $\mathrm{N}$ & 550 & 312 & 862 \\
\hline \multirow[t]{4}{*}{ Ocoña } & $a$ & -11.22 & -10.07 & -11.28 \\
\hline & $b$ & 3.23 & 2.93 & 3.23 \\
\hline & $\mathrm{R}^{2}$ & 0.95 & 0.96 & 0.96 \\
\hline & $\mathrm{N}$ & 788 & 627 & 1415 \\
\hline \multirow[t]{4}{*}{ Majes } & $a$ & -10.85 & -9.97 & -11.00 \\
\hline & $b$ & 3.15 & 2.90 & 3.17 \\
\hline & $\mathrm{R}^{2}$ & 0.95 & 0.96 & 0.94 \\
\hline & $\mathrm{N}$ & 864 & 996 & 1860 \\
\hline
\end{tabular}

a: intercepto, b: coeficiente de crecimiento relativo, $R^{2}$ : coeficiente de determinación, $\mathrm{N}$ : número de ejemplares

\section{Relación Longitud - Peso Total}

Según los resultados (Cuadro 4), el coeficiente de determinación $\left(\mathrm{R}^{2}\right)$, en general, fue $\geq 0.94$. El coeficiente de crecimiento relativo $b$ en los machos fue superior respecto al de las hembras en los ríos Tambo, Ocoña y Majes, más notorio en los dos últimos. Esto muestra que, el crecimiento relativo en los machos es mayor en peso que en talla en los ríos mencionados. Solo en el río Cañete, hubo proximidad de $b$ en machos y hembras.

\section{Índices Poblacionales}

El análisis por estrato altitudinal (Figura 4A) muestra que las mayores densidades medias fueron 1.47 ind. $/ \mathrm{m}^{2}$ en el río Cañete, 0.69 ind.$/ \mathrm{m}^{2}$ en el Tambo, $0.95 \mathrm{ind} / \mathrm{m}^{2}$ en el Ocoña y 1.5 ind. $/ \mathrm{m}^{2}$ en Majes, siendo todos observados en el estrato de 01-100 msnm de cada río.

La densidad media en los ríos Cañete, Tambo y Ocoña disminuyó de manera pro- 

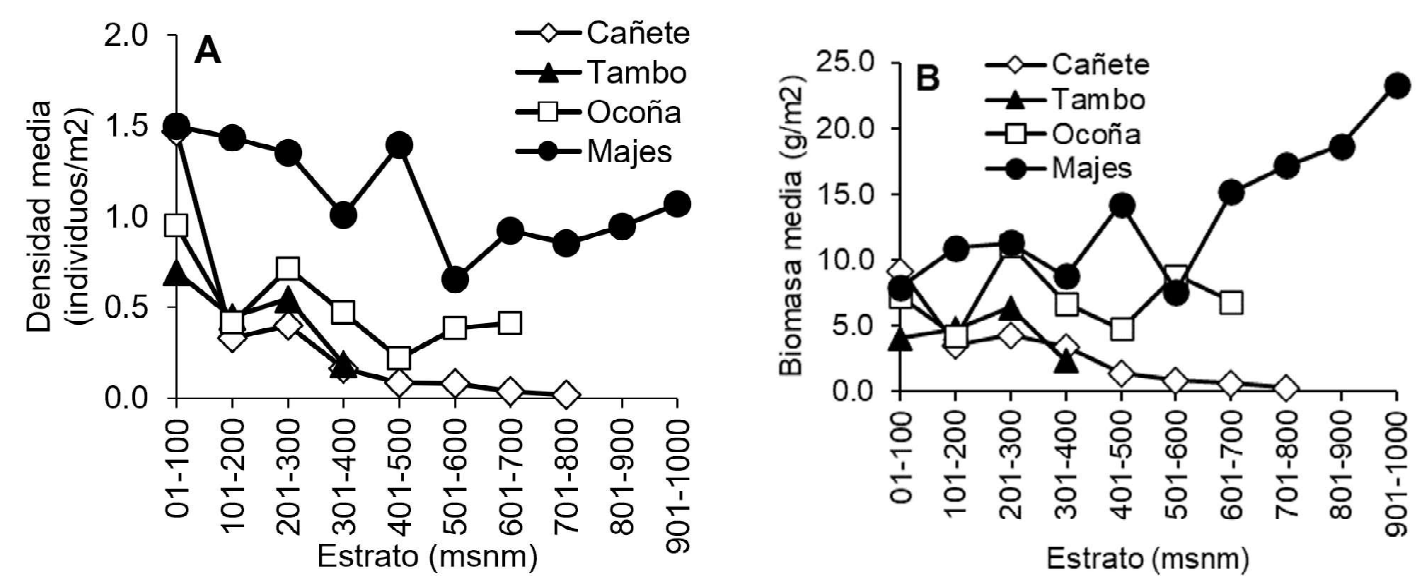

Figura 4. Variación altitudinal de los índices de densidad media (A) y biomasa media (B) de Cryphiops caementarius en los ríos del Perú

gresiva en los estratos más altos. En el río Majes, los valores fueron el evados $(>0.9$ ind. $\left./ \mathrm{m}^{2}\right)$ en los primeros cinco estratos y en el último, y bajos $\left(<0.9\right.$ ind. $\left.\mathrm{m}^{2}\right)$ en los estratos de 501 a $900 \mathrm{msnm}$. El río con mayor densidad media por estrato fue el Majes y el de menor fue el Tambo.

La biomasa media (Figura 4B), en el río Cañete varió de 0.30 a $9.17 \mathrm{~g} / \mathrm{m}^{2}$, superó los $3.0 \mathrm{~g} / \mathrm{m}^{2}$ en los estratos de 01 a $400 \mathrm{msnm}, \mathrm{y}$ fue inferior a $1.5 \mathrm{~g} / \mathrm{m}^{2}$ en altitudes mayores a $400 \mathrm{msnm}$. En el río Tambo varió de 2.32 a $6.37 \mathrm{~g} / \mathrm{m}^{2}$, el estrato de 201-300 msnm presentó mayor biomasa media. En el río Ocoña, varió de $4.23 \mathrm{~g} / \mathrm{m}^{2}$ (estrato 101-200 msnm) a $11.14 \mathrm{~g} / \mathrm{m}^{2}$ (estrato $201-300 \mathrm{msnm}$ ). En el río Majes varió de $7.63 \mathrm{~g} / \mathrm{m}^{2}$ (estrato 501-600 $\mathrm{msnm}$ ) a $23.36 \mathrm{~g} / \mathrm{m}^{2}$ del estrato más alto (901$1000 \mathrm{msnm}$ ), superó los $15.00 \mathrm{~g} / \mathrm{m}^{2}$ en los estratos de 601 a $1000 \mathrm{msnm}$, y fue inferior a dicho valor en los estratos de 01 a $600 \mathrm{msnm}$.

\section{Biomasa}

Los ríos de mayor biomasa de camarón fueron el Majes (64 286 $\pm 1671 \mathrm{~kg}$ ) seguido del Ocoña (44 $739 \pm 1969$ kg), y los de menor biomasa fueron el Cañete $(7482 \pm 801 \mathrm{~kg}$ ) y Tambo $(8229 \pm 1152 \mathrm{~kg})$ (Figura 5).

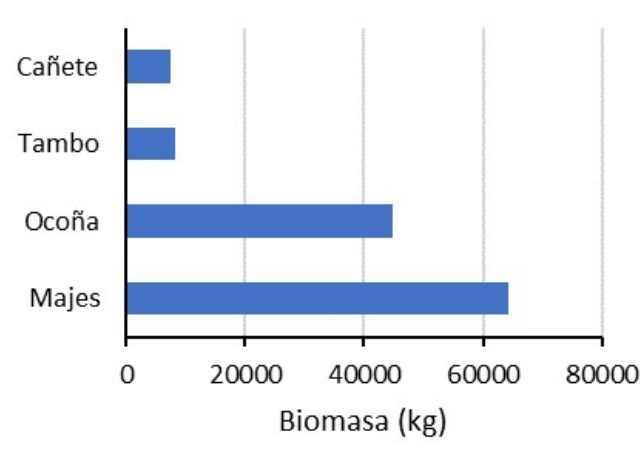

Figura 5. Biomasa estimada para Cryphiops caementarius en cuatro ríos de la costa peruana

\section{Relación Recurso - Ambiente}

Los coeficientes de correlación de Pearson entre la temperatura del agua y la biomasa en los ríos Cañete (0.79) y Tambo (0.32) evidencian que, la biomasa fue mayor en aguas más cálidas. También se observó mayor biomasa en aguas con moderados tenores $(8-10 \mathrm{mg} / \mathrm{l})$ de oxígeno disuelto en los ríos Tambo $(\mathrm{R}=-0.44)$, Ocoña $(\mathrm{R}=-0.46)$ y Majes $(\mathrm{R}=-0.36)$. Por otro lado, se observó correlación positiva entre la biomasa y la du- 
Cuadro 5. Coeficientes de Pearson de la correlación de parámetros del agua y biomasa del camarón de cuatro ríos de la costa peruana

\begin{tabular}{lcccc}
\hline Biomasa/río & Temperatura & Oxigeno & Dureza total & Cloro \\
\hline Cañete & 0.79 & 0.83 & -0.82 & 0.51 \\
Tambo & 0.32 & -0.44 & 0.22 & -0.39 \\
Ocoña & -0.69 & -0.49 & 0.52 & -0.84 \\
Majes & -0.57 & -0.36 & -0.51 & -0.33 \\
\hline
\end{tabular}

reza total en los ríos Tambo $(\mathrm{R}=0.22)$ y Ocoña $(\mathrm{R}=0.52)$. Finalmente, la correlación del cloruro con la biomasa fue negativa en los ríos Tambo $(\mathrm{R}=-0.39)$, Ocoña $(\mathrm{R}=-0.84)$ y Majes $(\mathrm{R}=-0.33)$, principalmente en los estratos bajos (Cuadro 5).

\section{Discusión}

Los ríos con condiciones adecuadas para el desarrollo de $C$. caementarius debieran presentar valores fisicoquímicos dentro de los siguientes rangos: temperatura 19 a $25^{\circ} \mathrm{C}$, pH 5 a 9, oxígeno disuelto 4 a 9 mg.L. $\mathrm{L}^{-1}$, bióxido de carbono 6 a $18 \mathrm{mg} . \mathrm{L}^{-1}$, alcalinidad total 50 a $250 \mathrm{mg} . \mathrm{L}^{-1}$ de $\mathrm{CaCO}_{3}$ y dureza total 20 a $280 \mathrm{mg} . \mathrm{L}^{-1}$ de $\mathrm{CaCO}_{3}$ (Llanos, 1980). Los resultados obtenidos en este estudio estuvieron dentro de los rangos aceptables para el normal desarrollo del camarón, similar a los reportado por Yépez y Bandín (1998), Wasiw y Yépez (2015), Zacarías y Yépez (2015). El incremento de la temperatura del agua hacia finales del año sería propio de la estación de primavera e inicio del verano, tal como lo observó también Llanos (1980) y García (2018). La presencia de cloro en el sector bajo de los ríos evaluados indica un grado de contaminación que debe ser objeto de mayor estudio. En este caso, su fuente estaría asociada a pesticidas utilizados en zonas agrícolas (Comisión Permanente del Pacífico Sur-
Programa de las Naciones Unidas para el Medio Ambiente [CPPS - PNUMA], 1997), lo cual muestra que el camarón tiene cierta resistencia a los actuales niveles de cloro.

Por otro lado, según Viacava et al. (1978), el camarón migra de las zonas bajas hacia las altas de los ríos en el transcurso de su vida y en el proceso van ganado talla como parte del crecimiento. Este comportamiento puede explicar lo observado en esta investigación, donde en los estratos más altos fueron más frecuentes ejemplares de gran porte, siendo esto más apreciable en los ríos Tambo, Ocoña y Majes. Los bajos valores en las tallas modales de los ríos Cañete, Tambo y Majes respecto a la talla modal del recurso en el río Ocoña evidencian una mayor presión sobre el recurso, los cuales podrían estar provocados, en parte por la pesca (Audzijonyte et al. (2013), pero también por consecuencia de una serie de factores, tales como la contaminación, modificación o destrucción del hábitat (Wasiw y Yépez, 2015).

El porcentaje inferior a $10 \%$ de camarones hembras en fases III y IV en el periodo de julio a diciembre es normal según el proceso de maduración del recurso, que desova con intensidad en los meses del verano (enero-marzo), tal como lo señalan Meruane et al. (2006) y Wasiw y Yépez (2015). 
Los machos alcanzaron mayores tamaños que las hembras y, por lo tanto, también mayor peso. Esto fue observado también por Viacava et al. (1978), que es común dado al dimorfismo sexual que presentan (Modesto, 1997). Sin embargo, en el río Cañete, tanto machos como hembras mostraron similar tendencia de su crecimiento relativo en talla y peso (Wasiw y Yépez, 2017), lo que en cierta forma evidencia que la población del camarón en este río está compuesta por ejemplares pequeños, $\mathrm{y}$ donde habría poco número de machos con tallas grandes, signo de sobreexplotación. Los valores del coeficiente de crecimiento relativo $(b)$ fueron en general superiores a 3.05, lo que muestra que el camarón presenta crecimiento alométrico positivo, tal como lo señaló Froese, (2006), Similares resultados obtuvieron también Wasiw y Yépez (2015).

La biomasa media en el río Majes incrementó con la altitud, observado también por Zacarías y Yépez (2015), y en contraste con los otros ríos evaluados, fue el que tuvo mayores niveles de biomasa media. La variación altitudinal de los índices de concentración está relacionada con el grado de acceso a las áreas de pesca, al nivel de la actividad extractiva y a la influencia de las actividades antrópicas como la construcción de estructuras en el río, desvío de las aguas con fines de agricultura, entre otros (Wasiw y Yépez, 2015).

La biomasa del camarón estimada en este estudio para el río Cañete fue ligeramente superior al reportado por IMARPE $(6461 \mathrm{~kg})$ en 2019, inferior para el caso del río Tambo (14 $403 \mathrm{~kg}$ ), relativamente similar con relación al río Ocoña (64 $298 \mathrm{~kg}$ ) y ligeramente inferior en el caso del río Majes (66204 kg).

Es importante conocer la relación recurso-ambiente para una adecuada gestión del recurso (Parsons et al., 2010; Ávila et $a l .$, 2015). En ese sentido, en el estudio se encontraron relaciones entre el oxígeno disuelto y la biomasa en el río Cañete, obser- vación reportada también por Wasiw y Yépez (2017), pero también entre la dureza y la biomasa, lo cual es importante siendo que el camarón desarrolla mejor en aguas ricas en carbonatos (Llanos, 1980).

La información de esta investigación es de valor para direccionar las estrategias de ordenación pesquera sostenible. Es necesario sensibilizar a los pescadores para que respeten la talla mínima de captura y así se pueda dar las condiciones para que la renovación de la población del recurso, principalmente en el río Cañete, donde el recurso muestra claros signos de sobreexplotación.

\section{Agradecimientos}

A Víctor Yépez Pinillos, Director General de Acuicultura del Instituto del Mar del Perú por el valioso aporte técnico-científico brindado para la elaboración del presente artículo.

\section{Literatura Citada}

1. [ANA] Autoridad Nacional del Agua. 2016. Protocolo nacional para el monitoreo de la calidad de los recursos hídricos superficiales. Lima, Perú: Industrial Alarcón. $88 \mathrm{p}$.

2. Avila H, García S, Rosas-Acevedo J. 2015. Análisis de componentes principales, como herramienta para interrelaciones entre variables fisicoquímicas y biológicas en un ecosistema léntico de Guerrero, México. Rev Iberoam Cienc 2: 43-53.

3. Audzijonyte A, Kuparinen A, Gorton $\boldsymbol{R}$, Fulton EA. 2013. Ecological consequences of body size decline in harvested fish species: positive feedback loops in trophic interactions amplify human impact. Biol Letters 9: 20121103. doi: 10.1098/rsbl.2012.1103

4. Baltazar P, Colán C. 2014. Algunos aspectos biológicos pesqueros de Cryphiops caementarius «camarón de rio» 
(Molina, 1782) en la cuenca baja del río Mala. Científica 11: 30-45. doi: 10.21142/ cient.v11i1.183

5. Csirke J. 1980. Introducción a la dinámica de poblaciones de peces. FAO Documento Técnico de Pesca $\mathrm{N}^{\circ} 192$. Roma: FAO. 82 p.

6. CPPS-PNUMA 1997. Diagnóstico regional sobre actividades realizadas en tierra que afectan los ambientes marino, costero y dulceacuícolas asociados al Pacífico Sudeste. Borrador de Informe - Plan de acción para la protección del medio marino y áreas costeras del Pacífico Sudeste, Lima, Perú: Comisión Permanente del Pacífico Sur CPPS. 37 p.

7. Espino M, Wosnitza-Mendo C. 1984. Manuales de evaluación de peces $\mathrm{N}^{\circ}$ 1. Área barrida. Inf Instituto del Mar del Perú N. ${ }^{\circ}$ 86. Lima, Perú: IMARPE. 32 p.

8. Froese R. 2006. Cube law, condition factor and weight-length relationships: history, meta analysis and recommendations. J Appl Ichthyol 22: 241-253. doi: 10.1111/j.14390426.2006.00805.x

9. García A, Alonso JC, Carvajal F, Moreau J, Nuñez J, Renno JF, Tello $S$, et al. 2009. Life-history characteristics of the large Amazonian migratory catfish Brachyplatystoma rousseauxii in the Iquitos region, Peru. J Fish Biol 75: 2527-2551. doi: 10.1111/j.10958649.2009.02444.x

10. García J. 2018. Estudio hidrológico de las cuencas Camaná, Majes, Sihuas, Quilca - Vittor - Chili con información satelital. Tesis de Ingeniero Forestal. Lima, Perú: Univ. Nacional Agraria La Molina. $103 \mathrm{p}$.

11. [IMARPE] Instituto del Mar del Perú. 2019. Anuario científico tecnológico IMARPE. Enero-Diciembre 2018. Vol 18. Callao, Perú. 146 p.

12. Llanos J. 1980. Aspectos ambientales de algunos ríos camaroneros del Perú. Tesis Doctoral. Trujillo, Perú: Univ. Nacional de Trujillo. $46 \mathrm{p}$.
13. Meruane J, Morales M, Galleguillos C, Rivera M, Hosokawa H. 2006. Experiencias y resultados de investigaciones sobre el camarón de río del norte Cryphiops caementarius (Molina 1782) (Decapoda: Palaemonidae): historia natural y cultivo. Gayana (Concepción) 70: 280-292. doi: 10.4067/S0717-65382006000200015

14. Modesto J. 1997. Evaluación biológica del camarón de río (Cryphiops caementarius) en el río Sama con fines de protección. Tacna, Perú: Univ. Nacional Jorge Basadre Growmann. 20 p.

15. Morales MC, Meruane J. 2013. The northern river shrimp Cryphiops caementarius (Decapoda, Palaemonidae). Research chronology between 1958 and 2008, I: distribution, population status, biology and life cycle, Crustaceana 86: 1441-1451. doi: 10.1163/ 15685403-00003259

16. Parada C, Yannicelli B, Hormazábal $S$, Vásquez S, Porobice J, Ernst B, Gatica $C$, et al. 2013. Variabilidad ambiental y recursos pesqueros en el Pacífico suroriental: estado de la investigación y desafíos para el manejo pesquero. LatAm J Aquat Res 41: 1-28. doi: 103856/ vol41-issue1fulltext-1

17. Parsons A, Watmough S, Dillon P, Somers K. 2010. Relationships between lake water chemistry and benthic macroinvertebrates in the Athabasca Oil Sands Region. J Limnol 69(Suppl 1): 118125. doi: 10.3274/JL10-69-S1-12

18. Valle JJ, Kuroiwa JM. 2010. Field studies and proposed solutions to facilitate migration of the Peruvian shrimp (Cryphiops caementarius). In: World Environmental and Water Resources Congress 2010: Challenges of Change. Rhode Island, USA.

19. Viacava M, Aitken R, Llanos J. 1978. Estudio del camarón en el Perú 1975 1976. Bol Inst Mar Perú 3: 165-232.

20. Wasiw J, Yépez V. 2015. Evaluación poblacional del camarón Cryphiops caementarius en Ríos de la costa sur 
del Perú. Rev Inv Vet Perú 26: 166-181. doi: 10.15381/rivep.v26i2.11103

21. Wasiw J, Yépez V. 2017. Evolución de la condición poblacional del camarón Cryphiops caementarius en el río Cañete (2000-2015). Rev Inv Vet Perú 28: 13-32. doi: 10.15381/rivep.v28i1.12942

22. Yépez, V, Bandín R. 1996. Estimación poblacional del camarón Cryphiops caementarius Molina 1782 (Natantia, Palaemonidae) en los ríos Ocoña, Majes -
Camaná y Tambo. Inf Progr Inst Mar del Perú 43: 3-31.

23. Yépez V. 2009. Consideraciones acerca de la distribución y extracción del recurso «camarón» en ríos de la costa peruana. Pesca 101: 9-11.

24. Zacarías S, Yépez V. 2015. Camarón de río Cryphiops caementarius (Molina, 1782) en la costa centro-sur del Perú, 2007. Bol Inst Mar Perú 42: 398-415. 